Editor's Note: These short reviews of recent JNeurosci articles, written exclusively by students or postdoctoral fellows, summarize the important findings of the paper and provide additional insight and commentary. If the authors of the highlighted article have written a response to the Journal Club, the response can be found by viewing the Journal Club at www.jneurosci.org. For more information on the format, review process, and purpose of Journal Club articles, please see http://jneurosci.org/content/ preparing-manuscript\#journalclub.

\title{
A Dynamical System Framework for Theorizing Preparatory Inhibition
}

\author{
(1)Gerard Derosiere \\ Cognition and Actions Laboratory, Institute of Neuroscience, Université catholique de Louvain, Brussels, Belgium \\ Review of Hannah et al.
}

During action preparation, the primary motor cortex (M1) and the spinal cord display considerable changes in neural activity. The functional role of this preparatory activity has been questioned for decades, motivating many investigations, from transcranial magnetic stimulation (TMS) to single-neuron studies.

On the one hand, studies that have applied TMS over M1, eliciting motorevoked potentials (MEPs) in the electromyography recording of targeted muscles, have revealed a suppression of corticospinal excitability during action preparation (i.e., lower MEP amplitudes relative to baseline; Duque et al., 2017). To better understand the extent of this preparatory inhibition, neuroscientists have examined its spatial specificity within M1 by measuring its impact on MEPs elicited in different muscles. Preparatory inhibition was found to affect muscles that are selected for the forthcoming action (e.g., MEP suppression in a right index muscle before right index movements), muscles that are non-selected but are part of the potential effector repertoire (e.g., MEP suppression in a non-selected left index muscle before right index movements), or are even com-

Received Jan. 11, 2018; revised Feb. 20, 2018; accepted Feb. 23, 2018.

Correspondence should be addressed to Gerard Derosiere, Cognition and Actions Laboratory, Institute of Neuroscience, Université catholique de Louvain, Avenue Mounier, 53-Bte B1.53.04, 1200 Bruxelles, Belgium. E-mail: gerard.derosiere@uclouvain.be.

DOI:10.1523/JNEUROSCI.0069-18.2018

Copyright $\odot 2018$ the authors $\quad 0270-6474 / 18 / 383391-03 \$ 15.00 / 0$ pletely task irrelevant (e.g., MEP suppression in pinky muscles). These findings suggest that preparatory inhibition operates in a global way (Duque et al., 2010; Greenhouse et al., 2015; Quoilin and Derosiere, 2015).

On the other hand, single-neuron studies have revealed that different cells within a given population do not exhibit a single, global pattern of activity during action preparation, but instead display various and complex temporal patterns (Churchland et al., 2010). For instance, the firing rate of a neuron in a population may rise throughout the preparatory period, while the activity of another neuron in the same population could remain initially stable and then decline as movement execution draws nearer. Investigation of the covariational structure of these patterns led to the proposal that preparatory activity emerges from the interactions between differently tuned neurons and serves to settle the initial state of a dynamical system whose future evolution will give rise to movement (Churchland et al., 2012; Elsayed et al., 2016).

One prediction arising from the dynamical system framework is that preparatory inhibition should entail a patterned suppression, rather than a global, uniformed one (i.e., only a subset of the inputs to corticospinal cells should be suppressed during action preparation). Hannah et al. (2018) tested this hypothesis in a recent article published in The
Journal of Neuroscience. Instead of questioning the specificity of preparatory inhibition by considering MEPs in different muscles (i.e., as realized in former TMS studies; Duque et al., 2010; Greenhouse et al., 2015), the authors examined its selectivity in distinct subpopulations of neurons controlling these muscles.

To tackle this issue, Hannah et al. (2018) took advantage of the fact that TMS over M1 can activate distinct subsets of cortical neurons synapsing on corticospinal cells and producing descending volleys with different latencies [i.e., the early and late indirect (I)-waves; Long et al., 2017]. While MEPs measured with standard TMS techniques in former studies reflect the summation of these waves (together with direct-waves elicited by the direct stimulation of corticospinal cells; Duque et al., 2010; Greenhouse et al., 2015), Hannah et al. (2018) exploited a combination of innovative stimulation approaches to selectively probe the electromyographic signature of each descending volley [Hoffman (H)-reflex conditioning and directional TMS; see below]. Doing so, they reasoned, could tell us whether preparatory inhibition differently alters the activity of distinct synaptic inputs to corticospinal cells.

In a first experiment, median nerve stimulation (MNS) was exploited to evoke H-reflexes-a measure of spinal excitability-in a forearm muscle during the preparation of wrist movements. In some trials, H-reflexes were conditioned with 
subthreshold TMS pulses over M1. This method amplifies the H-reflex, because of the facilitatory influence of the I-waves on spinal motoneurons (Niemann et al., 2017). Using different latencies between MNS and TMS, Hannah et al. (2018) tested whether action preparation selectively suppresses the early I-waves (short latencies), the late I-waves (longer latencies), or both. In another set of experiments, TMS with either posterior-to-anterior (PA) or anterior-to-posterior (AP) current directions was applied to elicit MEPs in a finger muscle when it was selected, non-selected, or irrelevant in different tasks. Here again, the rationale for using opposite current directions is that it activates separate subpopulations, with PA inducing early I-waves, and AP evoking late I-waves (Di Lazarro et al., 2017).

The results indicate that a specific subpopulation, responsible for the generation of the late I-waves, is systematically suppressed during action preparation, while another one, producing the early I-waves, is unaffected in some conditions. First, conditioned H-reflexes exhibited a reduced facilitation at the end of the preparatory period, compared with the beginning of the trial. Importantly, this decreased facilitation was observed only when latencies between MNS and TMS were long, hence indicating a suppression of the late I-waves. Second, MEPs elicited with PA currents (inducing early I-waves) were suppressed only when probed in a task-irrelevant muscle, but not when the muscle was selected or non-selected in a choice reaction time task. Contrariwise, MEPs elicited with AP currents (inducing late I-waves) were suppressed regardless of whether the muscle was selected, nonselected, or task irrelevant. Hannah et al. (2018) conclude that action preparation can involve the suppression of a selective subset of synaptic inputs to corticospinal cells and, thus, argue in favor of a dynamical system view of preparatory inhibition.

Exploiting the $\mathrm{H}$-reflex conditioning technique to disentangle the substrates of preparatory inhibition is highly innovative. Usually, researchers in the field rely on MEP amplitudes, which reflect the coarse summation of facilitatory and inhibitory processes occurring at both the cortical and spinal levels (Bestmann and Duque, 2016). Nonetheless, one should remain cautious when interpreting the results obtained with this technique. The fact that decreased facilitation of conditioned H-reflex was observed solely with long latencies between MNS and TMS certainly indicates that only the late
I-waves were altered by the experimental manipulations (Niemann et al., 2017). That said, it is important to note that this reduced facilitation could result from (1) a suppression of the late I-waves following the preparatory period (as proposed by the authors), (2) a facilitation of the late I-waves at the beginning of the trial, or (3) a combination of suppression and facilitation. Put differently, we cannot certify that facilitation of the conditioned $\mathrm{H}$-reflex was not boosted at the beginning of the trial compared with baseline, and that the decreased facilitation observed at the end of the preparatory period did not represent a return to baseline values. In fact, the conditioned H-reflex values observed during action preparation resemble those obtained at rest in previous studies using similar stimulation parameters (i.e., the conditioned H-reflex is $40-$ $50 \%$ larger than the unconditioned one; Taube et al., 2015). Hence, while the methodological approach adopted by Hannah et al. (2018) should inspire researchers in the field for designing future studies, those should include a baseline, resting-state evaluation of the conditioned $\mathrm{H}$-reflex to limit any potential confound in the interpretation of the results.

The findings obtained with directional TMS corroborate the conclusion of a selective suppression of the subpopulation generating the late I-waves, with no alteration of the subpopulation producing the early I-waves in some conditions. What would be the functional difference between these subpopulations? Interestingly, some studies have provided evidence that early I-waves are altered by increases in cortical excitability (provoked by the realization of a voluntary contraction), but not by increased inhibitory activity (induced by the administration of drugs that activate GABA receptors), while late I-waves are modulated by both (Di Lazzaro et al., 2017). Hence, early I-waves likely originate primarily from excitatory projections to corticospinal cells, while the generation of late I-waves likely involves both excitatory and inhibitory neurons. An implication of this is that PA and AP current directions may not allow the investigation of discrete subpopulations, but rather overlapping ones (i.e., the excitatory pools may overlap; the same argument holds true for the different latencies exploited in the H-reflex conditioning experiment). A second consequence is that a suppression of the early I-waves would essentially reflect a decrease of excitatory inputs to corticospinal cells, while a suppression of the late I-waves could reflect a decrease of excitatory inputs, an increase of inhibitory inputs, or both. Hence, the selective suppression of the late I-waves in the selected and non-selected muscles during the choice reaction time task (with no clear suppression of the early I-waves) suggest a specific increase of inhibitory inputs (with no decrease of excitatory inputs) to the corticospinal cells controlling these muscles. Conversely, the fact that the early and the late I-waves were suppressed to the same extent when the muscle was task irrelevant (Hannah et al., 2018, their Fig. $4 B$, superimposed PA and AP traces) may indicate that, in this case, corticospinal suppression was caused by a decrease of excitatory inputs (i.e., because early I-waves were suppressed), but did not involve any additional increase of inhibitory inputs (i.e., because the suppression of the late I-waves was not stronger than the suppression of the early I-waves). These interpretations illustrate the benefits of exploiting directional TMS to decipher the neural substrates of preparatory inhibition.

An important contribution of Hannah et al. (2018) is the proposal to conceptualize preparatory inhibition, as assessed with TMS, based on the dynamical system framework. So far, this framework has been exclusively exploited to theorize about the function of preparatory activity, as probed in a given population, through single-cell recordings (Shenoy et al., 2013). A central tenet of the theory is that the dynamics of preparatory activity in a population emerge as a result of the interconnectivity of the underlying cells (Gallego et al., 2017). The transposition of the framework to TMS investigations implies that this principle may apply for higher, more macroscopic levels of organization. In fact, the temporal dynamics of preparatory inhibition may be constrained by the interconnectivity of the different subpopulations within a given motor representation (i.e., within the representation of a given finger muscle), and of the motor representations themselves within M1. The issue of the dynamics of preparatory inhibition has not been investigated to date and may represent an interesting line of research for future studies.

To conclude, the work by Hannah et al. (2018) builds on timely questions regarding the selectivity and function of preparatory inhibition. Using two innovative techniques (H-reflex conditioning and directional TMS), Hannah et al. (2018) show that synaptic inputs to corticospinal cells present a patterned suppression during action preparation. On this basis, and 
in accordance with previous studies, the present Journal Club article discusses the strengths and weaknesses of the techniques, as well as the functional significance and the theoretical bearings of the findings. The present study paves the way toward a novel approach for conceptualizing and exploring the nature of preparatory inhibition, as assessed through TMS, derived from the dynamical system framework developed in the single-neuron literature.

\section{References}

Bestmann S, Duque J (2016) Transcranial magnetic stimulation: decomposing the processes underlying action preparation. Neuroscientist 22:392-405. CrossRef Medline

Churchland MM, Cunningham JP, Kaufman MT, Ryu SI, Shenoy KV (2010) Cortical preparatory activity: representation of movement or first $\operatorname{cog}$ in a dynamical machine? Neuron 68: 387-400. CrossRef Medline

Churchland MM, Cunningham JP, Kaufman MT, Foster JD, Nuyujukian P, Ryu SI, Shenoy KV
(2012) Neural population dynamics during reaching. Nature 487:51-56. CrossRef Medline

Di Lazzaro V, Rothwell J, Capogna M (2017) Noninvasive stimulation of the human brain: activation of multiple cortical circuits. Neuroscientist. Advance online publication. Retrieved March 5, 2017. CrossRef Medline

Duque J, Greenhouse I, Labruna L, Ivry RB (2017) Physiological markers of motor inhibition during human behavior. Trends Neurosci 40: 219-236. CrossRef Medline

Duque J, Lew D, Mazzocchio R, Olivier E, Ivry RB (2010) Evidence for two concurrent inhibitory mechanisms during response preparation. J Neurosci 30:3793-3802. CrossRef Medline

Elsayed GF, Lara AH, Kaufman MT, Churchland MM, Cunningham JP (2016) Reorganization between preparatory and movement population responses in motor cortex. Nat Commun 7:13239. CrossRef Medline

Gallego JA, Perich MG, Miller LE, Solla SA (2017) Neural manifolds for the control of movement. Neuron 94:978-984. CrossRef Medline

Greenhouse I, Sias A, Labruna L, Ivry RB (2015) Nonspecific inhibition of the motor system during response preparation. J Neurosci 35: 10675-10684. CrossRef Medline
Hannah R, Cavanagh SE, Tremblay S, Simeoni S, Rothwell JC (2018) Selective suppression of local interneuron circuits in human motor cortex contributes to movement preparation. J Neurosci 38:1264-1276. CrossRef Medline

Long J, Federico P, Perez MA (2017) A novel cortical target to enhance hand motor output in humans with spinal cord injury. Brain 140: 1619-1632. CrossRef Medline

Niemann N, Wiegel P, Kurz A, Rothwell JC, Leukel C (2017) Assessing TMS-induced D-and I-waves with spinal H-reflexes. J Neurophysiol. Advance online publication. Retrieved March 5, 2018. CrossRef Medline

Quoilin C, Derosiere G (2015) Global and specific motor inhibitory mechanisms during action preparation. J Neurosci 35:16297-16299. CrossRef Medline

Shenoy KV, Sahani M, Churchland MM (2013) Cortical control of arm movements: a dynamical systems perspective. Annu Rev Neurosci 36:337-359. CrossRef Medline

Taube W, Leukel C, Nielsen JB, Lundbye-Jensen J (2015) Repetitive activation of the corticospinal pathway by means of rTMS may reduce the efficiency of corticomotoneuronal synapses. Cereb Cortex 25:1629-1637. CrossRef Medline 